\title{
Etnobotánica del Ù-sulế, la casa cónica de los bribris en la comunidad Kácha'bli, Talamanca, Costa Rica
}

\author{
Sandra Paola Pozo-García ${ }^{1}$ (D) , Carlos O. Morales ${ }^{2}$ (D), \& Alí García Segura ${ }^{3}$ \\ 1. Universidad Estatal Amazónica, Facultad Ciencias de la Vida, Centro de Investigación, Posgrado y Conservación \\ Amazónica (CIPCA), provincia Pastaza, Ecuador. Universidad de Costa Rica, Sede de Occidente, Programa de Maestría \\ en Desarrollo Sostenible, Costa Rica; sp.pozog@uea.edu.ec; ppozog@hotmail.com \\ 2. Escuela de Biología, Universidad de Costa Rica, 11501-2060, San José, Costa Rica; carlos.moralessanchez@ucr.ac.cr \\ 3. Escuela de Filología, Lingüística y Literatura, Universidad de Costa Rica. Clan Sébaliwak, indígena bribri. San José de \\ Costa Rica. Costa Rica; sorbulu@gmail.com
}

Recibido 08-XI-2019 • Corregido 29-I-2020 • Aceptado 13-II-2020 DOI: https://doi.org/10.22458/urj.v12i1.2840

\begin{abstract}
Ethnobotany of the construction of a traditional ceremonial house (Ü-sulế) in a Bribri community of Talamanca, Costa Rica.". Introduction: The Bribri are an Amerindian group from Costa Rica that has a traditional house, the Ü-sulé, used for ceremonies led by the healer or Awá. This study was a shared experience about the Ù-sulế, with inhabitants of the Bribri Indigenous Territory in the communities of Kácha'bli, Korbita and Amu'bli in Talamanca, Costa Rica. Objectives: To describe the construction of an Uे-sulé, to observe and identify the plant species used throughout the process, to relate ethnobotanical information to cultural and historical aspects and to know some information about how this construction has changed in the past decades. Methods: Visits, meetings and interviews were organized in the aforementioned communities; all the stages of the construction were observed, and in a forest of the indigenous territory, specimens of the majority of plant species used in the process were collected and prepared. All the information for this study was provided by doctors (Awapa), older women, young people and indigenous Bribri professionals, who diligently collaborated to shape the study. Most plant species were identified by experienced curators from three Costa Rican herbaria (CR, JVR and USJ). Results: The complex construction process of an Ü-sulê is described in detail and for the first time the majority of plant species that are currently used to build this house were observed, collected and identified; amongst them Chloroleucon sp. (Fabaceae), Geonoma congesta and Iriartea deltoidea (both Arecaceae), Heteropsis oblongifolia (Araceae), three Miconia species (Melastomataceae), Minquartia guianensis (Olacaceae), and Pithecoctenium crucigerum (Bignoniaceae) are outstanding. Some species of plants used in the past were replaced because they had become scarce, or they were substituted against the ancestral wisdom (Siwã̃') in favor of a modern pragmatism related to criteria of sustainability. Discussion: The historical and cultural meaning of rescuing the almost lost tradition of Ü-sulé, which in the past was built by volunteer workers with the Bribri mentality of "we help each other", is discussed, but nowadays it is an onerous work carried out with business
\end{abstract}

RESUMEN. Introducción: Los bribris son un grupo amerindio de Costa Rica que tiene una casa tradicional, el Ù-sulê, utilizada para ceremonias dirigidas por el curandero o Awá. Este estudio fue una experiencia compartida sobre los Ù-sulế, con habitantes del Territorio Indígena Bribri en las comunidades de Kácha'bli, Korbita y Amu'bli en Talamanca, Costa Rica. Objetivos: Describir la construcción de un Ù-sulé, observar e identificar las especies de plantas utilizadas en todo el proceso, relacionar la información etnobotánica con aspectos culturales e históricos y conocer algunos datos sobre cómo ha cambiado tal construcción en las últimas décadas. Métodos: Se organizaron visitas, reuniones y entrevistas en las comunidades citadas; se observaron todas las etapas de la construcción, y en un bosque del territorio indígena se recolectaron y prepararon especímenes de la mayoría de especies de plantas utilizadas en el proceso. Toda la información para este estudio fue proporcionada por médicos (Awapa), mujeres mayores, jóvenes y profesionales indígenas bribris, que diligentemente colaboraron para dar forma al estudio. La mayoría de especies de plantas fueron identificadas por curadores experimentados de tres herbarios costarricenses (CR, JVR y USJ). Resultados: Se describe detalladamente el proceso complejo de construcción de un Ù-sulé y por primera vez se observaron, recolectaron e identificaron la mayoría de especies de plantas que actualmente se usan para construir esta casa; entre éstas se destacan Chloroleucon sp. (Fabaceae), Geonoma congesta e Iriartea deltoidea (ambas Arecaceae), Heteropsis oblongifolia (Araceae), tres especies de Miconia (Melastomataceae), Minquartia guianensis (Olacaceae) y Pithecoctenium crucigerum (Bignoniaceae). Algunas especies de plantas usadas en el pasado fueron sustituidas por haberse vuelto escasas, o bien se sustituyeron en contra de la sabiduría ancestral (Sĩwã') a favor de un pragmatismo moderno relacionado con criterios de sostenibilidad. Discusión: Se discute el significado histórico y cultural de rescatar la tradición casi perdida del Ù-sulé, que en el pasado era construido por trabajadores voluntarios con la mentalidad bribri de "nos ayudamos entre todos", pero actualmente es una obra 
mechanisms, influenced by the dominant Western culture. Thus, the monetization of this and other processes implies a risk for the traditional Bribri culture.

Keywords: Useful plant species, Bribri indigenous territory, Bribri-Cabécar indigenous culture, aboriginal wisdom (Sĩwã'). onerosa llevada a cabo con mecanismos empresariales, por influencia de la cultura occidental dominante. Así, la monetización de este y otros procesos implica un riesgo para la cultura bribri tradicional.

Palabras clave: Plantas útiles, territorio indígena Bribri, cultura indígena bribri-cabécar, sabiduría ancestral (Siñ̃̃').

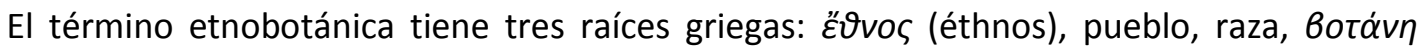
(botane), hierba, planta, + iké, estudio. Se trata del estudio del uso de las plantas por los pueblos tradicionales y de las relaciones recíprocas entre plantas y grupos humanos. Es una rama de la etnobiología. Aunque etnobotánica es un neologismo del siglo XIX, acuñado en 1895 por John W. Harshberger (Camacho-Zamora, 1997), realmente desde los inicios de la humanidad cada clan, tribu y sociedad sin duda tuvo una o más personas que, por ensayo y error, buscaron nuevos alimentos, materiales diversos, colorantes, medicamentos y venenos a partir de plantas. De modo predecible o demostrable, las zonas de mayor diversidad vegetal (ejs., cuenca mediterránea, sur de Asia y América tropical) ofrecieron las mejores posibilidades para experimentar.

La etnobotánica es, también, una rama muy importante de la botánica. No obstante, ni siquiera se menciona en la mayoría de textos de botánica. En general, ha recibido mayor atención de otros profesionales, tales como antropólogos y etnólogos, quienes normalmente se concentran en la dimensión social, cultural y espiritual de los pueblos, sin relacionarse directamente con los métodos ni los alcances de las ciencias naturales. Esto significa que su objeto de estudio no son las plantas por sí mismas ni las sociedades humanas como tales, sino todos aquellos fenómenos que de una u otra manera las asocian (Millot, 1968, citado por Camacho-Zamora, 1997).

El uso de plantas en culturas exóticas fue observado por exploradores, comerciantes, misioneros, naturalistas, antropólogos y botánicos. Sus puntos de vista eran muy diversos; por tanto, era difícil establecer una disciplina científica per se. La tarea tradicional consistía en catalogar los usos de las plantas en sociedades tradicionales. Esta recopilación de datos con interés en un problema intelectual es el fundamento de una ciencia; ésta ha aportado descubrimientos que representan una contribución significativa al bienestar humano. Por ejemplo, se calcula que entre 25 y $50 \%$ de las medicinas modernas se derivan de productos naturales, que en muchos casos se usaron primero como medicinas o venenos tradicionales. Actualmente, una síntesis moderna de la etnobotánica debe ser necesariamente interdisciplinaria (Davis, 1991). Ya no es solamente la labor del botánico y del antropólogo; es necesario incorporar las ideas, las observaciones y la visión de mundo de todas las personas que puedan aportar conocimientos, especialmente de quienes todavía viven cerca de la naturaleza, sin contacto directo con la tecnocracia que domina el mundo actual.

En Costa Rica existen varias etnias indígenas que han logrado mantenerse en contacto directo con la naturaleza y la cultura ancestral. Uno de esos pueblos es el bribri, que sigue practicando una serie de rituales fuertemente ligados a la tierra y a sus elementos. La casa tradicional bribri, llamada Ù-sulế, es uno de esos elementos, reflejo de un asentamiento humano determinado que agrupa un gran número de significaciones simbólicas, como mitos, leyendas y rituales que, de modo similar a otros pueblos ancestrales, se han plasmado en formas arquitectónicas según códigos y referentes históricos (González \& González, 1989). A menudo se ha usado el nombre español palenque para lo que los bribris llaman Ù-sulé. Según el diccionario de la Real Academia Española (RAE, 2019), en Costa Rica un palenque es una "Vivienda de varias familias de indígenas", mientras en otros países latinoamericanos este vocablo tiene otros significados. En 
la tradición bribri el Ù-sulế no es la casa de varias familias, sino un sitio ceremonial para hacer rituales, como los de curación del Awá (médico tradicional).

En un estudio previo (Pozo-García, 2017) se generó una propuesta para formular los indicadores de sostenibilidad adecuados para los pueblos indígenas, tomando a la población bribri como caso, particularmente en las manifestaciones culturales que se observan en el proceso de construcción de la casa tradicional Ù-sulế. El sitio de estudio, la comunidad de Kácha'bli, es una localidad reconocida en toda Talamanca por su empeño y fuerza comunitaria dirigida al rescate de la cultura. Allí se concentra el mayor número de casas Ù-sulé, así como los Ù stếbla (expertos en la construcción tradicional) y todos los elementos históricos y culturales que giran alrededor de esta infraestructura tradicional bribri.

A lo largo del tiempo, numerosos proyectos gubernamentales y no gubernamentales fueron prácticamente impuestos desde fuera a los pueblos indígenas, con la premisa de que tales pueblos debían sacrificar su cultura e identidad, para poder beneficiarse con el desarrollo y, de este modo, integrarse en la economía y la sociedad del resto de la nación (Deruyttere, 1997; Martínez-Castillo, 2004). Incluso algunas organizaciones, entre ellas universidades, se sienten como salvadoras de los pueblos indígenas y así desarrollan iniciativas sin haber consultado, siquiera, a los propios indígenas (Rojas-Morales, 2019). Sin embargo, sabemos que, por ejemplo, en la construcción de un Ù-sulế se manifiesta una gama de tradiciones y costumbres, que van desde la selección del tiempo apropiado, la preparación de las herramientas para la corta de los materiales necesarios y la selección de las especies de plantas, hasta la culminación e inauguración de la obra con una serie de rituales, que los indígenas aseguran fueron mandados por su héroe cultural Sibö' en el Sĩwã', que es como un libro oral sagrado de la sabiduría ancestral. Sobre este tema se ha investigado y escrito muy poco; esto es crítico porque, como los mismos indígenas reconocieron en las conversaciones para este estudio, ciertas tradiciones importantes tienden a abandonarse o se han abandonado del todo, en algunos casos sin que el mundo moderno llegara a conocerlas detalladamente. Por eso en la actualidad existen iniciativas de algunas comunidades indígenas costarricenses, para investigar, rescatar y poner en práctica tradiciones que forman parte de la sabiduría ancestral de estos pueblos autóctonos.

El abordaje de este estudio se ejecutó según el pensamiento bribri, incluyendo los fundamentos del marco metodológico, que fueron creados con base en patrones de comportamiento propios de su cultura. Una de las técnicas que más resultados ofrecieron fue la convivencia real y participativa con las comunidades, que permitió reconocer saberes propios de los pueblos indígenas. Taylor \& Bogdan (1987) señalan que los observadores participantes deben aprender a identificarse con los interlocutores, así como compartir con ellos(as) sus experiencias, sufrimientos y alegrías.

En vista de que prácticamente no se ha publicado información detallada sobre los aspectos etnobotánicos relacionados con la construcción de la casa tradicional bribri, el objetivo general fue describir el proceso constructivo actual del Ù-sulé en el Territorio Bribri de Talamanca. Los objetivos específicos fueron: a) Preparar especímenes de las plantas utilizadas en la construcción del Ù-sulé; b) Identificar las especies de plantas utilizadas; c) Interpretar el uso tradicional de materiales vegetales frente al uso actual de otras especies de plantas no tradicionales en el Ù-sulê. 


\section{MATERIALES Y MÉTODOS}

Área de estudio: El estudio se desarrolló en Costa Rica, provincia de Limón, cantón Talamanca, en la comunidad de Kácha'bli del Territorio Indígena Bribri. Debido a actividades culturales realizadas en Kácha'bli con influencia en comunidades aledañas y por relaciones de clanes, también se involucraron indirectamente las comunidades de Amu'bli y Korbita. Kácha'bli (distrito Telire, $09^{\circ} 30^{\prime} 41^{\prime \prime} \mathrm{N}, 8^{\circ} 58^{\prime} 41^{\prime \prime} \mathrm{W}$ ) significa "zanja llena de achiotal" [achiotal es un sitio con plantas de achiote: Bixa orellana L. (Bixaceae)]. Amu'bli significa "sitio con escasez de pita" [pita es probablemente Agave sp. (Agavaceae), plantas cultivadas (no nativas en Talamanca) con hojas fibrosas, de importancia textil]; Korbita $\left(09^{\circ} 30^{\prime} 14^{\prime \prime} \mathrm{N}, 82^{\circ} 59^{\prime} 36^{\prime \prime} \mathrm{W}\right)$, por estar a orillas del río Korbi, significa "escasez de sardinas" (Kếkëpa Ignolio Nercis, com. pers., 2016). Amu’bli se ubica a $1 \mathrm{~km}$ de Kácha'bli (de sur a norte) y es la comunidad más poblada, con características que tienden a lo urbano. El territorio Bribri de Talamanca alberga a 7772 habitantes y su extensión es de 66419 has. La zona agrícola (ca. 12000 has) la conforman las fincas del valle y unas pocas al pie de monte; las actividades de ganadería se ubican en las partes más altas (ca. 5000 has); el resto del territorio son zonas boscosas poco habitadas (Borge \& Castillo, 1997). Los bribris y cabécares, dos pueblos indígenas fuertemente relacionados en Talamanca, habitan, utilizan y dominan un extenso territorio de casi 200 mil has, otorgadas a ellos legalmente como reservas indígenas (Martínez-Castillo, 2004). Las asociaciones de desarrollo son los órganos administrativos que manejan las políticas y ejecutan proyectos en estas comunidades.

Diseño de investigación: Se optó por un diseño de investigación de tipo etnográfico, que parte de un enfoque cualitativo de perspectiva fenomenológica. Este método no solo se fundamenta en las bases que describen la lógica formal de pensamiento, sino que además está dotado de una correspondencia con la naturaleza extrínseca e intrínseca del fenómeno observado. Se aplicó una técnica, denominada en este estudio "lectura vivencial", que proviene de un concepto bribri: Prestar la mano; una forma tradicional de trabajo en conjunto que incluye todas las actividades de una comunidad para su bien, basada en el conocimiento que se obtiene al convivir durante un tiempo con la comunidad y compartir con ella un trabajo. De esta manera se comprendió mejor la cultura de los bribris y ciertos patrones que se repiten cuando se relacionan con su entorno y con otra gente; estos patrones se observaron en toda la comunidad y se manifestaron de modo deliberado en la construcción de una vivienda, ceremonias funerarias, la caza, la pesca y en actividades cotidianas. Se aplicaron dos niveles de interpretación: a) comunicacional: cambio de términos técnicos a un lenguaje sencillo; b) análogo: hallazgo de correspondencias tanto en patrones indirectos como en la aplicación de algunos principios de la sabiduría bribri.

Visitas y reuniones: Se planearon e hicieron visitas a médicos y mujeres, así como a líderes comunitarios, directamente en sus hogares. El punto de partida de cada conversación fue siempre el Ù-sulê, su historia, su tradición, su construcción y su importancia como actividad tradicional específica. En seguida se preguntó por indicadores particulares que explicaran de modo dinámico cómo se concibe un Ù-sulê en la tradición y en la cosmología bribri y, en consecuencia, cómo se debe construir y cuáles son los materiales que deben usarse. En estos diálogos siempre se buscaron los términos bribris y españoles más apropiados, para que todos los interlocutores pudieran entenderse mutuamente. En las etapas finales del estudio se organizaron reuniones con los representantes de las asociaciones, líderes comunitarios, directores de escuelas y comunidad en general, para consultar a varias personas de diversas asociaciones y clanes indígenas su opinión acerca de indicadores del Ù-sulế. La técnica de reuniones y conversatorios se planteó para definir, por consenso, la caracterización, construcción y evaluación de los indicadores para saber si un Ùsulé está primero bien planeado y después bien construido. Además, se acataron directrices dadas en un momento por el Awá y luego transmitidas por el I sãũkbla (líder de la construcción) y el Ù 
stébla, para que la presencia de investigadores y su participación no afectaran el desenvolvimiento y la integridad cultural de la actividad. A partir de estas reuniones, basándose en el permiso de la autoridad ancestral (Awá), los constructores acordaron la participación de la autora en la planificación de todas las actividades, la recolecta de materiales en el bosque, los rituales, la construcción en sí, la preparación de los alimentos y bebidas y hasta la narración de historias. Más detalles sobre visitas y reuniones se hallan en Pozo-García (2017).

Colección de muestras botánicas: Hubo un interés especial en las plantas utilizadas en la construcción del Ù-sulé, con la finalidad de: a) identificar las especies con su nombre científico correcto; b) conocer el estado de conservación de esas especies en Costa Rica (Libro Rojo de Especies Amenazadas de UICN); c) aportar especímenes de herbario con información etnobotánica valiosa; d) aportar información para estudios etnobotánicos comparativos del país. Durante las visitas, sábados y domingos fueron aprovechados para recolectar, tomar fotos y vídeos de las especies de plantas utilizadas en la construcción. La primera recolecta de especímenes se realizó en octubre de 2014. Los especímenes recolectados se herborizaron en el campo, ante jóvenes y mayores de una asociación local (Datsồkàta) para explicar el proceso de recolecta, preparación y conservación de estas muestras. Posteriormente, con la aprobación de la comunidad, las muestras se trasladaron al Herbario de la Universidad de Costa Rica (USJ), donde se completó el proceso de herborización. Los especímenes se identificaron no solamente en USJ, sino también en el Herbario Nacional de Costa Rica (CR) y en el Herbario de la Universidad Nacional de Costa Rica (JVR), con la colaboración de varios curadores en cada herbario. Algunas especies de Aráceas fueron identificadas mediante fotos por M.H. Grayum, del Herbario del Jardín Botánico de Misuri (MO). Además de los especímenes recolectados, que fueron depositados en USJ, se entregó a este herbario una base de datos sobre los especímenes con los siguientes campos de información: a) datos taxonómicos; b) datos ecogeográficos; c) datos para herborización; d) datos etnobotánicos; y e) datos auxiliares. La información etnobotánica especifica el uso de cada especie de planta al construir la casa tradicional Ù-sulếy sus propiedades, además de su nombre en bribri. Se anexó un archivo fotográfico ligado con códigos a la base de datos.

Vale aclarar que durante la recolecta de muestras la autora siempre fue acompañada por una persona local, para evitar comentarios o malos entendidos de los propietarios de las fincas. Cuando las fincas estaban ubicadas cerca del camino principal, donde era visible la presencia de visitantes, no se tuvo permiso para ingresar y hacer la recolecta. Por eso, solamente se recolectó en un bosque conservado de la finca del Kékëpa Rigoberto Gabb, cruzando el río Lari entre Kácha'bli y Coroma; con el permiso, el acompañamiento y la ayuda del señor Gabb allí se halló la mayoría de especies. Para llegar a la finca fue necesario cruzar a nado el río Lari, cargando todos los materiales de recolecta. Este cruce lo hacen los habitantes de algunos poblados indígenas para aprovisionarse de productos básicos en Amu'bli. Algunas personas han muerto arrastradas por la corriente caudalosa; cuando esto sucede, los indígenas lo interpretan según la cosmovisión bribri (Jara \& García, 2003; García, 2016; Durán, 2013): Los mayores dicen que un ser terrible llamado Dìnamu (tigre de agua) es el causante de las muertes en el río y agregan que no se construye un puente porque podría matar el espíritu del río.

Los resultados se describieron con base en los aportes que surgieron desde el pensamiento bribri, tanto respecto a la construcción en sí del Ù-sulé como a los indicadores para evaluar el proceso. Aquí vale aclarar que para esto se utilizó el sistema de indicadores de una correcta construcción propuesto por Pozo-García (2017); de esa obra seleccionamos usos y prácticas tradicionales relacionados con las especies de plantas $y$, en general, con la etnobotánica, conservando la numeración y el enunciado bribri original de los indicadores, tal como los establecieron los mismos indígenas. También aclaramos que los signos diacríticos y fonéticos del bribri se usan en este texto según el sistema de escritura de Jara \& García (2003), con énfasis en la 
versión fonética del bribri de Coroma.

Ética, conflicto de intereses y declaración de financiamiento: La autora y los coautores declaran haber cumplido con todos los requisitos éticos y legales pertinentes, tanto durante el estudio como en el manuscrito; que no hay conflictos de interés de ningún tipo, y que todas las fuentes financieras se detallan plena y claramente en la sección de agradecimientos. Asimismo, están de acuerdo con la versión editada final del documento. El respectivo documento legal firmado se encuentra en los archivos de la revista.

\section{RESULTADOS}

Se obtuvo mucha información valiosa a partir de un conjunto de historias y memorias en torno al tema Ù-sulế, que se narraron en ciertos momentos de la construcción y en las visitas realizadas a la comunidad. Así se recopilaron historias completas o fragmentos de ellas. Cada vez que se narraban estas tradiciones, el interlocutor ubicaba al receptor en el pasado, sobre todo "en tiempos de los mayores", probablemente hace más de cinco décadas (Pozo-García, 2017).

Especies de plantas: Se recolectaron 46 muestras botánicas y cuatro muestras auxiliares. La mayoría se identificó a nivel de género y especie y, en la base de datos, todas tienen un registro fotográfico. Dos especies no fueron recolectadas; la muestra de otra fue descartada, pero en ambos casos se cuenta con el registro fotográfico. El total de las muestras y fotografías abarcan 16 especies de plantas utilizadas en la casa cónica Ù-sulé (Cuadro 1), identificadas con nombre científico, nombre común o local y nombre bribri. Los usos etnobotánicos indicados se refieren solamente a la construcción de la vivienda tradicional, de modo que las mismas especies podrían tener otros usos en las comunidades bribris.

Las formas de crecimiento o hábitos de las especies son diversos. Así, tenemos que el $26 \%$ son árboles con alturas de 8 a $30 \mathrm{~m}$. Hay dos tipos de palmas (Arecaceae) representadas: una pequeña, de sotobosque, con tallos múltiples (ùkö o suita, Geonoma congesta), cuyas hojas se usan para tejer las esteras del techo; otra de tallo único, firme y robusto (jira, Iriartea deltoidea), de cuyo fuste se obtienen regletas que se usan como base de los aros y las costillas. Debido a la flexibilidad de esas regletas, estos son los elementos que le confieren a la construcción formas circulares. Se usan tallos de bejucos leñosos como tsika' (Pithecoctenium crucigerum) y raíces de bejucos trepadores o epífitos, como së'miñakicha (Philodendron alliodorum); incluso se usa la corteza de algunos árboles, como ulë'tklö (Trema integerrima). En la mayoría de los casos se prefiere usar la especie en un estado juvenil, en vista de que los tamaños y las propiedades físicas son apropiados para suplir las necesidades de estructura y función, que aseguran la durabilidad de la casa tradicional Ù-sulé.

Todas las especies identificadas fueron revisadas en la Lista Roja de Especies Amenazadas de UICN (https://www.iucnredlist.org), teniendo como resultado que la mayoría pertenece a la categoría "no evaluada" (NE). El árbol maderable Minquartia guianensis (manú o tsulë, Olacaceae) aparece categorizado como "casi amenazado (near threatened, NT); es decir, por su uso constante está cerca de calificar como amenazado en el futuro cercano. La especie arbórea Vitex cooperi (wìpi) se registra "en peligro" (endangered, EN); aunque no se halla en estado crítico, es propensa a estarlo. Chloroleucon sp. (cachá), por ser una especie desconocida, probablemente nueva, no ha sido registrada en la lista roja. Actualmente los bribris ya no usan las dos últimas especies en la construcción; han sido reemplazadas por manú, que se emplea en los postes principales, en vista de que confiere a la construcción más firmeza y durabilidad que las especies usadas anteriormente. 


\section{CUADRO 1}

Principales especies de plantas usadas en la construcción de la casa tradicional bribri Ù-sulë*

\begin{tabular}{|c|c|c|c|c|c|c|}
\hline Nombre científico & Familia & $\begin{array}{l}\text { Nombre } \\
\text { bribri }\end{array}$ & $\begin{array}{l}\text { Nombre } \\
\text { local o } \\
\text { nacional }\end{array}$ & Hábito & Parte usada & Uso en el Ù-sulë́ \\
\hline $\begin{array}{l}\text { Casearia arborea (Rich.) } \\
\text { Urb. }\end{array}$ & Salicaceae & yềtsi kalò & palo rollizo & Árbol & $\begin{array}{l}\text { Tronco } \\
\text { inmaduro }\end{array}$ & $\begin{array}{l}\text { Rollizos o vigas que forman el cono que } \\
\text { soporta aros, costillas y esteras. }\end{array}$ \\
\hline $\begin{array}{l}\text { Chloroleucon sp. A (apud } \\
\text { Zamora, 2010) }\end{array}$ & Fabaceae & (desconocido) & cachá & Árbol & Tronco & $\begin{array}{l}\text { Los bribris consideran que son } 2 \\
\text { especies diferentes: Una se usaba en } \\
\text { tiempos antiguos para los } 8 \text { postes } \\
\text { principales; otra se sustituye ahora por } \\
\text { manú, que tiene madera más resistente. }\end{array}$ \\
\hline $\begin{array}{l}\text { Geonoma congesta } \\
\text { H.Wendl. ex Spruce }\end{array}$ & Arecaceae & ùkö & suita & $\begin{array}{c}\text { Palma de } \\
\text { sotobosque }\end{array}$ & Hojas & $\begin{array}{l}\text { Para tejer las esteras que cubren el } \\
\text { techado. }\end{array}$ \\
\hline $\begin{array}{l}\text { Heteropsis oblongifolia } \\
\text { Kunth }\end{array}$ & Araceae & kốkicha & $\begin{array}{l}\text { tripa de pollo. } \\
\text { Nacional: } \\
\text { bejuco del } \\
\text { hombre }\end{array}$ & $\begin{array}{l}\text { Bejuco } \\
\text { trepador }\end{array}$ & Raíces aéreas & $\begin{array}{l}\text { Para amarrar estructuras, como rollizos } \\
\text { y costillas, y sujetar esteras al techo. }\end{array}$ \\
\hline $\begin{array}{l}\text { Iriartea deltoidea } \\
\text { Ruiz \& Pav. }\end{array}$ & Arecaceae & ãlầklö̀, õlõ & jira & Palmera & $\begin{array}{c}\text { Trozas } \\
\text { longitudinales } \\
\text { del tronco }\end{array}$ & $\begin{array}{l}\text { Tronco trozado como base de los aros y } \\
\text { como costillas (guía circular para la } \\
\text { puesta de palos rollizos y esteras). }\end{array}$ \\
\hline Miconia prasina (Sw.) DC. & Melastomataceae & $\begin{array}{l}\text { shuli } \\
\text { sarùrù }\end{array}$ & palo rollizo & Árbol & $\begin{array}{l}\text { Tronco } \\
\text { inmaduro }\end{array}$ & $\begin{array}{l}\text { Rollizos o vigas que forman el cono que } \\
\text { soporta aros, costillas y esteras. }\end{array}$ \\
\hline $\begin{array}{l}\text { Miconia punctata (Desr.) } \\
\text { D. Don ex DC. }\end{array}$ & Melastomataceae & shuli mãt & palo rollizo & Árbol & $\begin{array}{l}\text { Tronco } \\
\text { inmaduro }\end{array}$ & $\begin{array}{l}\text { Rollizos o vigas que forman el cono que } \\
\text { soporta aros, costillas y esteras. }\end{array}$ \\
\hline $\begin{array}{l}\text { Miconia smaragdina } \\
\text { Naudin }\end{array}$ & Melastomataceae & $\begin{array}{l}\text { shuli } \\
\text { sarùrù }\end{array}$ & palo rollizo & Árbol & $\begin{array}{l}\text { Tronco } \\
\text { inmaduro }\end{array}$ & $\begin{array}{l}\text { Rollizos o vigas que forman el cono que } \\
\text { soporta aros, costillas y esteras. }\end{array}$ \\
\hline $\begin{array}{l}\text { Minquartia guianensis } \\
\text { Aubl. }\end{array}$ & Olacaceae & tsulë & manú & Árbol & Tronco & Para los 8 postes principales. \\
\hline $\begin{array}{l}\text { Mucuna holtonii } \\
\text { (Kuntze) Moldenke }\end{array}$ & Fabaceae & dulëkkicha & ojo de buey & $\begin{array}{l}\text { Bejuco } \\
\text { trepador }\end{array}$ & Tallo maduro & $\begin{array}{l}\text { Bejuco grueso que sujeta y envuelve los } \\
\text { aros y la corona de la casa tradicional } \\
\text { bribri. }\end{array}$ \\
\hline $\begin{array}{l}\text { Philodendron } \\
\text { alliodorum Croat \& } \\
\text { Grayum }\end{array}$ & Araceae & sềmiñakicha & bejuco negro & $\begin{array}{l}\text { Bejuco } \\
\text { trepador }\end{array}$ & Raíces aéreas & Para tejer esteras para el techo. \\
\hline $\begin{array}{l}\text { Philodendron } \\
\text { radiatum Schott }\end{array}$ & Araceae & tlaña & (desconocido) & Epífita & Raíces aéreas & $\begin{array}{l}\text { Para hacer amarras y tejer esteras en } \\
\text { reemplazo de sềmiñakicha, para el } \\
\text { techo. }\end{array}$ \\
\hline $\begin{array}{l}\text { Pithecoctenium } \\
\text { crucigerum } \\
\text { (L.) A. Gentry }\end{array}$ & Bignoniaceae & tsika' & $\begin{array}{l}\text { (desconocido) } \\
\text { Nacional: } \\
\text { bateíta }\end{array}$ & $\begin{array}{l}\text { Bejuco } \\
\text { trepador }\end{array}$ & $\begin{array}{c}\text { Tallo } \\
\text { inmaduro }\end{array}$ & $\begin{array}{l}\text { Para sujetar los } 3 \text { aros que van sobre los } \\
\text { postes. }\end{array}$ \\
\hline Tetracera sp. & Dilleniaceae & kaë kalò & pata de pava & $\begin{array}{l}\text { Bejuco } \\
\text { leñoso }\end{array}$ & $\begin{array}{c}\text { Tallo } \\
\text { inmaduro }\end{array}$ & $\begin{array}{l}\text { Para sujetar los } 3 \text { aros que van sobre los } \\
\text { postes. }\end{array}$ \\
\hline $\begin{array}{l}\text { Trema integerrima } \\
\text { (Beurl.) Standl. }\end{array}$ & Cannabaceae & sul, ulët́klö & (desconocido) & Árbol & $\begin{array}{l}\text { Corteza } \\
\text { fibrosa }\end{array}$ & $\begin{array}{l}\text { Se hacen tiras delgadas y largas para } \\
\text { tejer esteras para el techo. }\end{array}$ \\
\hline Vitex cooperi Standl. & Lamiaceae & wìpi & plátano & Árbol & Tronco & $\begin{array}{l}\text { Para los } 8 \text { postes principales. } \\
\text { Actualmente es reemplazado por manú. }\end{array}$ \\
\hline
\end{tabular}

Fuente: Base de datos de los especímenes de plantas (Pozo-García, 2017). Recolectores: Paola Pozo y Rigoberto Gabb.

Las muestras fueron determinadas en los herbarios USJ, CR y JVR. Se depositaron en el herbario USJ.

* Ver el apéndice digital 1 
Indicadores de la casa ceremonial Ù-sulế. Los indicadores de una correcta construcción tradicional del Ù-sulé suman 33 y se ordenaron según las fases tradicionales del proceso constructivo (PozoGarcía, 2017). En la última fase se agregaron indicadores sugeridos por varios líderes indígenas y representantes de las asociaciones, que no describen el proceso constructivo en sí, sino aspectos fundamentales de la tradición y las relaciones con la comunidad. En general, los puntos en que la mayoría coincide sobre la correcta construcción de un Ù-sulế, que guardan relación con la etnobotánica, son los siguientes:

1. Selección del terreno ("Aquí es": ĩ uuirö). Un Ù-sulế se construye en un lugar bien plano, sin pendientes y más arriba que otras viviendas, sin charcos ni barro cerca. El terreno no puede tener la tierra rajada ni estar en riesgo por inundaciones, movimientos sísmicos o fuertes ráfagas de viento (evidentes por la presencia de árboles caídos o quebrados en dirección al sitio de la construcción); la dirección del viento debe ser hacia la puerta del Ù-sulé, es decir, de este a oeste.

2. Limpieza y descanso de la tierra (Ù blàñõ wéke). Cuando el lugar elegido para construir la casa tiene vegetación herbácea y arbustiva (terreno abandonado o ruderal), se dice que se halla en estado impuro; por eso es necesario limpiar, se amontona el material y se quema. El Awá hace el ritual de limpieza y lo entrega al constructor. El terreno limpio se deja descansar por un día, se hace ayuno y al siguiente día ya está puro. Entonces hay que evitar fumar, tomar cualquier bebida o comer dentro de esta área por todo el tiempo que dure la construcción.

3. Curación de herramientas y materiales por el Awá (I kéli enã i tsé wồike awá tö). Antes de salir a cortar los materiales necesarios, el Awá debe curar las herramientas que se van a usar, que deben ser solo las tradicionales (machete y hacha).

4. Antes de la corta, se guarda discreción y se usa lenguaje correcto (I péñe). El líder de la construcción debe recordarlo a los trabajadores participantes. Esto se hace para despistar a los dueños de los materiales, para que no los oculten. Además, es importante guardar silencio en el bosque ("la montaña") para no molestar a los dueños y evitar accidentes.

5. Corta en buena luna ( téke si' wa). Solamente en tiempo de Luna buena (bribri si'tuí: Luna oscura), que es el Cuarto Menguante, se pueden cortar todos los árboles, bejucos y hojas. Este tiempo dura una semana.

6. Corta con buena mano (Ulà batse'). Los trabajadores que van al bosque a cortar los materiales no pueden ser mujeres en período de menstruación o embarazadas. Los esposos que tengan a su esposa en este estado tampoco podrán participar.

7. Lo que hay en nuestra tierra hay que respetarlo (Se'kốwöta íyi tso'skốs, Seuekốwötã ötã tso'skốs seoukốkĩ e' dlồiök). Ser prudente con el tiempo durante la corta y evitar el desperdicio. Los materiales que se calculan desde el inicio deben ser suficientes; se corta solo lo que se necesita; no se puede salir a cortar otra vez, porque eso indicaría error de cálculo. La corta se debe realizar dos a tres días antes de iniciar la construcción. Después de la corta, todos los materiales deben estar tapados por un día con hojas de bijagua blanca (Calathea sp., Marantaceae) y solamente un Awá puede destaparlos.

8. Piso de tierra y limpieza ( ké páiklök shkít). El piso del Ù-sulế siempre será de tierra y circular. Este espacio tiene símbolos relativos a la creación de la Tierra y al origen de los bribris.

9. Tiene ocho postes (/ sè dökã páköl). Los postes que dan forma circular a la base del Ù-sulé son ocho de wìpi (Vitex cooperi), más dos que sostienen el cobertizo en la entrada. Los postes deben tener un hueco en el extremo superior para incrustar el primer aro y deben estar un poco inclinados hacia fuera y ser todos iguales. No se usan herramientas modernas, como motosierras, niveladores y cintas métricas. Si van a usarse postes de manú (Minquartia guianensis), antes debe hacerse ayuno. 
10. El Ù-sulế tiene una sola puerta (I wékö ét chë) hacia el Este, donde nace el Sol. Los postes de la puerta deben estar bien ubicados.

11. Tipos de rollizos y forma de plantar (I kéli). Los palos rollizos deben ser de yềtsi kalò (Casearia arborea), shùlí sarùrù (Miconia prasina) y shùlí mãt (Miconia punctata) (Cuadro 1), entre otras especies nombradas en lengua bribri, que no pudieron ser identificadas. Para plantar conservando la forma circular se marca un círculo, tomando como referencia el centro; luego, para corregir que los rollizos estén bien plantados se usa jira, chonta o caña blanca rajada [ $u k a^{\prime}$, caña brava: Gynerium sagittatum (Aubl.) P. Beauv., Poaceae], y este material se cruza de un palo rollizo a otro. El amarre de los rollizos debe estar en la cúspide. Este material debe estar fresco para que sea flexible; así se podrá formar el círculo y ver fácilmente si está mal plantado en algún punto.

12. Cuenta con tres aros ( $/$ bchi' dlö mañat tö kicha). La presencia de tres aros es fundamental, porque estos darán lugar a cuatro capas y cada una tiene su significado.

13. Tipos de bejucos para aros ( $/$ tsé $i$ bchi' muố). Para amarrar el aro tradicionalmente se usaba shtökicha, naman, kaë kalö (Tetracera sp.) y tsika' (Pithecoctenium crucigerum). Las dos primeras especies no se observaron en este estudio y, por tanto, no pudieron ser identificadas. El naman es un bejuco grande que por su dureza se usaba para construir puentes de hamaca; ya no se usa, en gran parte porque es difícil encontrarlo. En la construcción actual, observada, se usaron algunos bejucos tradicionales y también dulëkkicha (Mucuna holtonii), pero este último bejuco es recomendable solo para trabajos temporales, no de larga duración.

14. Bejuco blanco para amarras ( $/$ tsé $i$ kéli muố kốkicha). El bejuco usado para amarrar las costillas es kốkicha (Heteropsis oblongifolia). Si escasea, se usa tlaña (Philodendron radiatum) o la corteza del árbol kapôli. No pudimos ver esta última especie, pero Jara \& García (2003) anotan que es una laurácea medicinal, usada en rituales de purificación de las mujeres en estado ña (de embarazo). Para sujetar los rollizos con los aros siempre se usa kốkicha. No pueden usarse clavos.

15. Poste central del Ù-sulé ( $/$ tse' míke ù shứshõ e', e tso choklu). El poste central de la construcción se retira y es reemplazado por un bejuco de kókicha que queda colgando desde la cúspide, donde divide y simboliza el centro del mundo.

16. Las amarras de bejuco deben recordar las "estrellas" del mundo (I tsé ta kor, i tsé batà e'rök bkuwö). Las costillas se amarran y las esteras se pegan con kókicha. Las estrellas se forman dejando los extremos de las amarras libres hacia adentro de la casa. Todo palo rollizo debe tener al menos una estrella.

17. El cobertizo o culata inclinada (/ lè) de la puerta se hace con el mismo material del techo. La culata debe ser inclinada y estar pegada al techado cónico, a tres metros del suelo. La inclinación deber ser moderada.

18. Hoja de suita (Geonoma congesta) para el techado (ùkö). Las esteras deben tejerse solo con hojas de esta palma. Así, este material no tiene reemplazo.

19. Bejuco negro para tejer esteras (sềmiñakicha). El bejuco sềmiñakicha (Philodendron alliodorum) se usa tradicionalmente; si escasea es reemplazado por el bejuco tlaña, la corteza del árbol sul (= ulë'tklö: Trema integerrima) o corteza del bejuco dulëkkicha (Mucuna holtonii).

20. Las esteras de la cúspide se tejen sobre bejuco ( $/$ tsồ pàbkók). La cúspide del techo se cubre con seis "esteras de corona"; es decir, las esteras se tejen sobre un bejuco grueso para poder envolverlas y van acortándose para cubrir el ápice del techo cónico.

21. Tres tucas de leña en el centro (Böukuö ù shứshõ e'). No se colocan cuatro o cinco tucas de leña en el fogón, sino solamente tres.

22. Ritual de quema de sobros (Ù bla ña wèke). Los materiales sobrantes se pueden reutilizar y se guardan de modo ordenado. Los que no se reutilizan se juntan y se colocan en el centro del Ù-sulë; después el Awá hace un ritual y los quema junto con plantas de dipà (chile: Capsicum sp., Solanaceae), como un agradecimiento a los dueños de la montaña (del bosque), con el humo 
devolviendo lo prestado.

25. Presencia de hamacas (Kapò̀ kchàtchố). Para la inauguración se colocan varias hamacas; al menos cuatro. La hamaca debe ser tejida de forma tradicional con fibras de amùkkõ (Musa sp., Musaceae, que también se usan para cargar canastas) o fibras del árbol tsaritkuo, no visto ni identificado en nuestro estudio.

\section{DISCUSIÓN}

Cada vez que los bribris narraron tradiciones del pasado, percibimos la fuerza de la tradición en este pueblo; no obstante, al mismo tiempo fue evidente que los tiempos modernos han cambiado, también para los indígenas, y que han puesto en peligro la sabiduría ancestral (Jara \& García, 2003).

En relación con el espacio vital en que se ha desarrollado su mundo, Palmer et al. (1992) citan el relato de la Kékeëpa Eustacia Palacios, quien dijo que Sibö' les indicó a los indígenas dónde podían cultivar la tierra y construir los ranchos. "Por eso sabemos que en el bosque virgen, donde hay árboles muy altos con troncos gruesos, no debemos cultivar". Es muy diferente el modo de actuar de los explotadores del bosque con mentalidad mercantil, porque siempre buscaron precisamente bosques y otros parajes vírgenes, para despojarlos de sus maderas finas y sus metales preciosos ( $c f$. Galeano, 1971). La cultura bribri tradicional no puede manifestarse ni sobrevivir sin el bosque, de donde ha obtenido la mayor parte de sus bienes.

El material botánico fue recolectado cerca de los sitios donde se hizo la corta para la construcción del Ù-sulế. Estos son bosques secundarios recuperados de largos períodos de monocultivos (banano y cacao), que ahora evidencian un notable estado de conservación. Allí se pueden encontrar ahora la mayoría de especies para la construcción tradicional. Sin embargo, algunas especies usadas tradicionalmente, como Philodendron alliodorum (Cuadro 1), un bejuco trepador, epífito y colgante que crece en árboles viejos y altos del bosque primario, no se encuentran en bosques secundarios y la gente "debe ir más lejos" para poder hallarlas. Detalles interesantes sobre el trabajo de corta en sí y el transporte de los materiales hacia el sitio de construcción, gran parte a hombros y espaldas de los indígenas, pueden leerse en Pozo-García (2017). La bijagua blanca (bribri mulùsik), que se usa para tapar todos los materiales para el Ù-sulé, después de ser cortados, es probablemente Calathea lutea (Aubl.) Schult. (Marantaceae), con el envés de las hojas glauco (casi blanco), pero Camacho- Zamora (1983) y Constenla-Umaña (2010) citan $C$. crotalifera $\mathrm{S}$. Watson ( $=C$. insignis Petersen). Es probable que se usen ambas especies o que exista un problema de identificación.

Es muy interesante la idea de economía de la naturaleza que tienen los indígenas bribris al señalar que se realiza una sola corta de todo y no se desperdicia nada. El desperdicio está relacionado con el principal pecado reconocido en la cultura bribri, la mezquindad (Bozzoli, 1979). Los bribris afirman que si se corta un árbol en la fase lunar de Cuarto Creciente, en tres a cuatro días se le caerán todas las hojas, se llenará de polillas y morirá. En cambio, cuando se corta en Cuarto Menguante, árboles, hojas y bejucos vuelven a crecer. La influencia de la Luna en el crecimiento de las plantas se mantiene como una sólida creencia en muchos pueblos indígenas y también en numerosas comunidades rurales del mundo (Flores, Meléndez, Luna \& González, 2012). Los factores que afectan el crecimiento de las plantas son complejos y variados, pero en algunas especies se ha observado que al aumentar la luminosidad de la Luna hacia la fase de Luna Llena, aumenta también el flujo de savia (Masé, 2017). Sin embargo, la ciencia occidental moderna no ha podido demostrar una clara relación directa entre fases lunares y crecimiento vegetal; los resultados varían con cada diseño experimental y cada especie estudiada. 
Un aspecto notable del pensamiento bribri es el respeto a los dueños de las especies del bosque, un concepto abstracto y espiritual que no existe en la mentalidad occidental. Así, cuando van a cortar plantas en el bosque, los indígenas procuran ser discretos y usar un lenguaje apropiado para los dueños. Según el mandato del Sĩwã', los bribris consideran que los elementos del bosque no son diferentes ni inferiores; tampoco son solo utilitarios. Animales y plantas se consideran verdaderamente vivos, con espíritu; "son otras semillas". A diferencia de las culturas judeocristianas occidentales (Hall, 2011), en la filosofía bribri no existe una jerarquía con seres dominantes arriba e inferiores abajo, sino una relación circular, que ha generado transferencias entre el estado del bosque, el modo de vida indígena y la cosmovisión de este pueblo. En este sentido es muy significativo un relato escuchado a los bribris (Awá Ricardo Morales y su hijo Julio Sánchez, com. pers., 2014, citados por Pozo-García, 2017): Para Sibö' lo que cuenta es el espíritu y la naturaleza; él le dijo al zopilote [una especie local de buitre]: "Usted es una buena persona y se quedará viviendo aquí...". También el espíritu del viento y de la tierra se quedaron aquí, porque Sibö' los calificó como personas buenas. Hall (2011) cita otros pueblos tradicionales, fuertemente apegados a la naturaleza, en que plantas, animales y hasta elementos abióticos son llamados personas, lo que muestra que, para esas culturas, los seres y elementos de la naturaleza son tan importantes como las personas humanas. En la película ruso-japonesa Dersú Uzalá, dirigida por Akira Kurosawa (1975), basada en hechos reales ocurridos durante las primeras expediciones rusas en el extremo oriental de Siberia, el aborigen siberiano que guió a los exploradores rusos les dijo que los ríos caudalosos y los vientos fuertes son personas malas. Hall (2011) cita al aborigen australiano Bill Neidjie, para quien un humilde pasto, igual que un hermoso árbol, son como nuestros hermanos y padres.

Otro aspecto sorprendente de la cosmovisión indígena bribri es que consideran importante el ayuno mientras dura la construcción dentro del círculo de la planta del Ù-sulế; allí no debe haber comida ni bebida. También si se usan postes de manú (Minquartia guianensis) hay que ayunar, en vista de que este árbol es sagrado por estar ligado directamente con rituales funerarios: En la tradición bribri el manú se usa para construir el camastro de madera en que se coloca el cuerpo del difunto. Ellos consideran que si no se ayuna al usar manú, la casa se pudrirá pronto. Este trabajo tan importante debe hacerse como lo indicó Sibö'. Además, dada la importancia cultural e histórica de esta infraestructura, un Ù-sulế no puede construirse sin la presencia de un Awá (médico), que observa que todo el proceso se realice según la tradición y los mandatos del Sĩwã'; solamente el Awá puede hacer los rituales, porque conoce profundamente las historias y los significados de cada parte de la casa.

Entre las especies de plantas identificadas, una es un árbol fabáceo notable, porque todavía no posee nombre específico, aparece como "Chloroleucon sp. A" en el Manual de Plantas de Costa Rica (Zamora, 2010) y probablemente es una especie nueva. Es un hecho notable que los bribris hayan usado, durante cientos o miles de años, una especie fabácea que los botánicos no conocían, en uno de los países botánicamente más explorados de la región neotropical. Al mismo tiempo, vale aclarar que perdura el problema de que los indígenas ven dos especies, donde los botánicos ven una sola de Chloroleucon ( $c f$. Cuadro 1). Sobre los usos de las plantas identificadas, es muy probable que ningún botánico haya registrado hasta ahora que los indígenas bribris usan las especies melastomatáceas identificadas (Miconia prasina, M. punctata y M. smaragdina) como palos rollizos o vigas para sostener grandes estructuras. Aparentemente eso era posible solo con especies de maderas muy duras (ejs., fabáceas, sapotáceas, lauráceas, etc.), pero no con melastomatáceas, que en su mayoría son árboles pioneros con maderas suaves. De hecho, se usan los troncos inmaduros de estas melastomatáceas, que son rectos y más bien flexibles, pero resistentes. Para sustituir los palos rollizos de antaño se ha sugerido hasta la posibilidad de usar eucaliptos cultivados (Eucalyptus spp., Myrtaceae), nativos de Australia, con crecimiento rápido y troncos rectos; pero algunos 
señores (Kékëpa) indican que de esta manera se perdería la tradición del Sĩwã'. En todo caso, la tradición nunca contempla el uso de especies introducidas.

Vale aclarar que en este estudio la especie de palmera llamada jira es Iriartea deltoidea; sin embargo, esta especie es muy similar a, y a menudo se confunde con Socratea exorrhiza (Mart.) H. Wendl. (chonta), que probablemente se ha usado en el Ù-sulé igual que la primera. Del mismo modo, la palma de sotobosque llamada suita ha sido identificada en este estudio como Geonoma congesta, pero es probable que también se use otra especie con hojas algo similares llamada igualmente suita: Asterogyne martiana (H. Wendl.) H. Wendl. ex Hemsl. (Arecaceae), de amplio uso para techar en comunidades indígenas de bajuras muy lluviosas. Otra especie similar a la anterior, que también habita en Talamanca, es Geonoma cuneata H. Wendl. ex Spruce (Grayum, 2003). Futuros investigadores de la cultura bribri podrán dilucidar este asunto. La suita para techar el $\dot{U}$ sulé no se puede cultivar; crece silvestre en sitios sombreados del bosque.

Se han usado trozas de troncos de palmas como chonta o jira (Iriartea deltoidea) para reemplazar el palo de bchi' (indeterminado) o la caña brava (Gynerium sagittatum), materiales que tienen la propiedad de ser flexibles y se usaban para dar las formas circulares a toda vivienda tradicional. También los cabécares, vecinos de los bribris, usan la caña brava (= blanca) para construir paredes de habitaciones (Camacho-Zamora, 1983). Al pasar el tiempo aumentó la densidad de población, la jira empezó a escasear y ya no había suficientes palmeras en el valle para construir nuevas casas ni reparar las viejas. Para reparar las casas, los habitantes de Talamanca empezaron a usar hojas de cinc; esto se hizo común ya en la década de 1970. Estas hojas metálicas se compraban a comerciantes y llegaban por ferrocarril; pese a ser más caras, las preferían porque duraban más tiempo que los materiales tradicionales obtenidos en el bosque (Golliher, 1977). Los más apegados a la tradición sustituyeron jira, cachá y wìpi por manú, que se caracteriza por su dureza y resistencia, y su dureza es sinónimo de fuerza (Jara \& García, 2003). Cuando se usaba cachá o wìpi no había posibilidad de reutilizar el material; con el manú sí se puede. La duración de los materiales usados en tiempos de los mayores con el tratamiento tradicional era de tres años, mientras que el manú dura más de 10 años sin tratamiento y puede reutilizarse en otras construcciones. Este cambio de uso ocurrió hace más de 20 años (Kékëpa Hernán García y Rigoberto Gabb, comun. pers., 2015), porque los nuevos materiales duran más tiempo y es más fácil encontrarlos, pero de esta manera no se respetaron las normas del Sĩwã'. En tiempos de los mayores abundaba el cachá, según algunos indígenas. Tanto cachá como wìpi son especies escasas; consiguientemente, si se usaran constantemente para construir, éstas y otras especies se volverían más escasas y hasta podrían extinguirse localmente. Además, era necesario curar las maderas de ambas especies antes de ser insertadas en el suelo, para que no se deterioraran rápidamente; una práctica tradicional abandonada, que actualmente solo se encuentra en las memorias de los mayores. Esto representa una pérdida cultural y un cambio notable en las dinámicas de la construcción.

Los bejucos, que para muchas personas no indígenas (síkua) son plantas extrañas e insignificantes, como de segunda o tercera categoría en el reino vegetal, para los bribris tienen gran utilidad y profundos significados. Para amarrar usan a menudo las raíces resistentes de bejucos y epífitas; de allí el vocablo bribri kichà (raíz) (Constenla-Umaña, 2010) en los nombres de varias especies de bejucos. Además, una cuerda de kókicha (Heteropsis oblongifolia), que debe colgar desde la cúspide en el centro del Ù-sulế, según los bribris es para su defensa, porque este bejuco engaña a las enfermedades y así los protege. Ese bejuco puede usarse para sostener ollas o canastas para aprovechar el fogón, pero no se permite jugar con él. Para tejer esteras se usó tradicionalmente el bejuco sềmiñakicha (Philodendron alliodorum). Sin embargo, según los expertos bribris los materiales que lo sustituyen en la actualidad [el bejuco tlaña (Philodendron radiatum), la corteza del árbol sul (Trema integerrima) o corteza del bejuco dulëkkicha (Mucuna holtonii)] no son 
apropiados en un Ù-sulé, porque son de duración más corta; por eso el dulëkkicha se usaba antes solo para eventos culturales de duración corta. Cuando se usa tlaña para tejer esteras, solo dura tres días y por eso no se recomienda para la casa. En algunas esteras se han usado incluso fibras de nailon, un material sintético comprado en ferreterías, que como otros se encuentra más fácilmente y es más barato. Antes se usaron las ramas duras de sotacaballo [Zygia longifolia (Humb. \& Bonpl. ex Willd.) Britton \& Rose, Fabaceae] como ganchos para sostener la estera para la corona de la cúspide.

González \& González (1989) citan al Kékëpa Mario Nercis, quien señaló que la gente puede enfermarse por usar materiales que no son los tradicionales. Cambiar los materiales indicados en el Sĩwã' se considera una muestra de pereza y desobediencia a la sabiduría ancestral; sin embargo, se justifica solamente cuando hay escasez. El uso de materiales más duraderos para construir el Ù-sulế es positivo bajo el prisma de principios modernos de sostenibilidad. Sustituir madera poco resistente por otra que dura el doble de tiempo parece un cambio significativo, puesto que postes, rollizos, vigas y bejucos deben sostener toda la casa. Además, poder reutilizar materiales es otro punto favorable. Así, vemos que tiende a imperar un criterio económico, que implica un cambio de valores de lo tradicional a lo sostenible.

Los bribris indican que el fogón central con tres tucas de leña debe estar humeando todos los días, para que la construcción se impermeabilice, se combata la humedad de forma natural y se ahuyenten del Ù-sulé los bichos, tales como insectos (ejs. comejenes y hormigas), arañas y escorpiones. Para eso debe usarse leña apropiada, que además sirva para ahumar las hojas del techo y evitar insectos dañinos; por ej. trul, surimklö, tamaklö (guabo: Inga edulis Mart., Fabaceae), wàlo [Inga spectabilis (Vahl) Willd.] y kurklö [guarumo: Cecropia sp., Cecropiaceae, que también se usa para confeccionar el báculo del Awá; Kékëpa Fausto Morales, com. pers., 2016]; las dos primeras especies no pudieron identificarse durante este estudio. Sobre la presencia de hamacas, Bozzoli (1979) cita a H. Pittier (1938), quien describió las fiestas dentro del Ù-sulé y afirmó que en el interior "se colgaban del techo un centenar de hamacas". Los niños no pueden sentarse en las hamacas puestas para los(as) señores(as) (Kékëpa). Las hamacas facilitan el espacio adecuado para las ceremonias del médico tradicional; cuando termina una ceremonia de curación, las hamacas se guardan.

Desde el punto de vista cultural, es interesante la ceremonia de inauguración del Ù-sulë, en la que se sirve la chicha tradicional Alä́chika y se prepara comida para todos los presentes. La chicha se hace con maíz molido sobre piedra, que se envuelve en una hoja de bijagua verde (probablemente Calathea sp., Marantaceae) y este bulto se llama mojoso. Las mujeres que participaron en la construcción preparan la comida; si se necesita ayuda, intervienen otras mujeres de la comunidad. La comida se sirve en hojas de kalsik [bijagua común: Calathea crotalifera: "material universal para envolver por sus hojas grandes" (Camacho-Zamora, 1983)] y suele ser carne de cerdo, carne de pollo casero, carne de res, banano (Musa acuminata Colla, Musaceae), yuca (Manihot esculenta Crantz, Euphorbiaceae) y palmito (varias especies, Arecaceae). Tanto la chicha como la comida se reparten a todos; aunque haya mucha, no se vende, sino que se regala. Según uno de los señores (Kếkëpa Albir Morales, citado por Pozo-García, 2017), en la inauguración no se permite bailar en el Ù-sulé con música síkua (de blancos) ni se permite el paso de turistas con comportamientos dudosos (como los hippies).

En el pasado el Ù-sulé y otras obras importantes se construían mediante un esfuerzo comunitario. González \& González (1989) citan una expresión bribri que lo resume: “Hoy se trabajó en tu campo; mañana en el mío"; así se pagaban las labores tradicionalmente. El intercambio de bienes materiales y trabajo era muy importante en la cultura tradicional; al respecto sobresalía la "mano vuelta" o "prestar mano", que era ayudarle a otro en un trabajo sin cobrarle, y el otro devolvía un trabajo proporcional al que se le hizo (Martínez-Castillo, 2004). Al final se preparaba 
una fiesta de chichada para todos los trabajadores. La tradición de laborar voluntariamente ("prestar mano") en la construcción de un Ü-sulế se perdió en los últimos 40 a 50 años. La posibilidad de construir y tener un Ù-sulé ya no está al alcance de cualquier miembro de la comunidad, como alguna vez en el pasado lo estuvo. Actualmente, solo las asociaciones pueden lograrlo tras conseguir suficiente apoyo económico y tener el respaldo de personas no indígenas con dinero y poder. También depende de tener un área de bosque, donde se puedan encontrar las plantas y los materiales necesarios. Esto fue reconocido por varios interlocutores.

La construcción de un Ù-sulé representa actualmente un costo mayor a siete millones de colones (ca. 11900 dólares de EE. UU.) (Pozo-García, 2017). El mayor porcentaje de gastos fue el pago de salarios a los trabajadores (78 \%), que cubrió el Ministerio de Trabajo del Gobierno de Costa Rica. La asociación que planeó y organizó el trabajo solo pudo cubrir un $3.33 \%$ del gasto total. De este modo se impuso una perspectiva empresarial, una visión del mundo totalmente opuesta a los principios indígenas. En tiempos de los mayores el Ù-sulé se usaba solamente para vivir la cultura o conocer el Sĩwã por medio del Awá. En la cosmología bribri el uso del Ù-sulế debe ser aceptado por Sibö'; por eso, otros usos no eran correctos. Sin embargo, la voluntad de la comunidad para rescatar sus tradiciones se ve claramente amenazada por la monetización de la cultura. En parte por esta razón en la actualidad se dan otros usos al Ù-sulé; uno de ellos es el demostrativo-educativo, con apoyo de algunas universidades, que integran en varias carreras giras de campo para que profesores y estudiantes visiten comunidades indígenas. La construcción de un Ù-sulé como casa ceremonial se perdió hace más de 80 años, pero se rescató siguiendo patrones no tradicionales. Así se trazó una ruta a seguir, más fácil y más eficaz, que procede de la cultura no indígena, pero atenta directamente contra la tradición.

"El Ù-sulé es un reflejo de la vida buena para todos". Esta expresión encierra un aspecto crucial de la mentalidad de los bribris y de las implicaciones de esta infraestructura para el desarrollo de la comunidad. Aunque la lengua bribri no posee un término equivalente a progreso, la búsqueda de la "vida buena para todos" es su forma de pensar y concebir el mundo; para lograrla, "entre todos nos ayudamos". Por eso el progreso bribri es el trabajo cooperativo de la comunidad, no de personas individuales. En la construcción del Ù-sulé se reconoce que hay una ciencia y unos especialistas que atesoran el conocimiento, porque se trata de una tarea ardua y complicada, que muy pocas personas pueden concebir, planear y realizar.

\section{AGRADECIMIENTOS}

En la identificación de los especímenes de plantas prestaron una colaboración valiosísima los siguientes curadores: Eduardo Chacón (USJ), Alexander Rodríguez (CR), Michael H. Grayum (MO), Luis J. Poveda (JVR) y Pablo Sánchez-Vindas (JVR). En las visitas a las comunidades indígenas, numerosas personas ofrecieron toda la información que hizo posible este estudio; a todas ellas expresamos nuestro respeto y agradecimiento, especialmente a los interlocutores con cargos tradicionales, quienes otorgaron el permiso espiritual para compartir su cultura y sabiduría: Awá Rafael Seies, Awá Ricardo Morales, Awá Terencio Campos y Óköm Fernando Waiza. De igual manera a los Kékëpa Fausto Morales y su esposa Demetria Sánchez; Rigoberto Gabb, Mario Nercis $(\dagger)$ y su esposa Gilbertina Sánchez; Albir Morales; Nemesio Reyes; Hernán García y a todos los "zopilotes constructores". Este artículo es uno de los resultados de la Tesis de Maestría de la autora (ver PozoGarcía, 2017), quien hace aquí patente su agradecimiento a la tutora Ma. Eugenia Bozzoli. Dedicamos este trabajo, con muestras de gran respeto y admiración, a la memoria del Kékëpa Mario Nercis (30 de agosto 2018), quién se identificó a tal nivel con el estudio, que logró construir de forma conjunta - el bribri y el sikua, como lo hicieron Sibö y Ogma en las historias bribris- una adecuada interpretación de los indicadores para el Ù-sulé. Su aporte fue relevante en la comprensión de la 
forma de pensar bribri; del mismo modo, su ausencia representa para Costa Rica una pérdida de saberes ancestrales y la filosofía indígena de un sabio con un alto nivel ético y un gran conocimiento.

\section{REFERENCIAS}

Borge, C., \& Castillo, R. (1997). Cultura y conservación en la Talamanca indígena. San José, Costa Rica: EUNED.

Bozzoli, M. E. (1979). El nacimiento y la muerte entre los bribris. San José, Costa Rica: Editorial Universidad de Costa Rica.

Camacho-Zamora, J. A. (1983). Etnobotánica Cabécar. América Indígena, 43(1), 57-86.

Camacho-Zamora, J. A. (1997). Perspectiva histórica de la etnobotánica. Repertorio Americano, nueva época, 3, $20-31$.

Constenla-Umaña, A. (2010). Toponimia Bribri. Estudios de Lingüística Chibcha, 29, 109-132.

Davis, W. (1991). Towards a new synthesis in ethnobotany. In Ríos, M. \& Pedersen, H.B. (Eds.), Las plantas y el hombre. Memorias del Primer Simposio Ecuatoriano de Etnobotánica y Botánica Económica (pp. 339-357). Quito, Ecuador: Ediciones Abya-Yala.

Deruyttere, A. (1997). Pueblos indígenas y desarrollo sostenible: El papel del Banco Interamericano de Desarrollo. Presentación ante el Foro de las Américas. Washington, D.C., EE.UU: Unidad de Pueblos Indígenas y Desarrollo Comunitario, Departamento de Desarrollo Sostenible, Banco Interamericano de Desarrollo. Recuperado de https://publications.iadb.org/es/publicacion/15890/pueblos-indigenas-y-desarrollo-sostenible-el-papel-delbanco-interamericano-de

Durán, F. J. (2013). Esperando al tigre de agua: Los vigilantes insobornables del río. Portal Nacional de Biodiversidad, Museo Nacional de Costa Rica, San José, Costa Rica. Recuperado de http://ecobiosis.museocostarica.go.cr/recursos/ZOOLOGIA/BiologiaCultural/Vigilantes\%20de\%20Dinamu/Vi gilantes\%20de\%20Dinamu.aspx

Flores, L., Meléndez, F., Luna, G., \& González, E. (2012). Influencia de las fases lunares sobre el crecimiento del maíz (Zea mays variedad NB6). Ciencia e Interculturalidad, 10(1), 132-148. DOI: 10.5377/rci.v10i1.819

Galeano, E. (1971). Las venas abiertas de América Latina. Buenos Aires, México: Siglo Veintiuno Editores.

García, A. (2016). Ditsồ rukuồ . Identidad de las semillas: Formación desde la naturaleza / Identity of the seeds: Learning from nature. Gland, Switzerland: IUCN.

Golliher, J. (1977). Casas comunales autóctonas en Talamanca, Costa Rica. Análisis etno-histórico. Vínculos, 2, 145-163.

González, A., \& González, F. (1989). La Casa Cósmica Talamanqueña y sus simbolismos. San José, Costa Rica: EUNED.

Grayum, M. H. (2003). Arecaceae. En Hammel, B. E., Grayum, M. H., Herrera, C., \& Zamora, N. (Eds.), Manual de Plantas de Costa Rica, vol. 2, Gimnospermas y Monocotiledóneas (Agavaceae-Musaceae) (pp. 201-293). St. Louis, EE.UU.: Missouri Botanical Garden Press.

Hall, M. (2011). Plants as Persons: A Philosophical Botany. New York, EE.UU.: Sunny Press.

Jara, C. V., \& García, A. (2003). Diccionario de Mitología Bribri. San José, Costa Rica: Editorial Universidad de Costa Rica.

Martínez-Castillo, R. (2004). La estructura cultural indígena en Talamanca. Praxis, 57, 37-48.

Masé, G. (2017). Science update: Moonlight [Can moonlight affect plant growth?]. Urban Moonshine. Recuperado de https://www.urbanmoonshine.com/blogs/blog/science-update-moonlight

Palmer, P., Sánchez, J., \& Mayorga, G. (1992). Vías de extinción, vías de supervivencia: Testimonios del pueblo indígena de la Reserva Kéköldi, Costa Rica. San José, Costa Rica: Editorial Universidad de Costa Rica. 
Pozo-García, S. P. (2017). Sostenibilidad en el proceso constructivo del Ù-sulé y las implicaciones en el desarrollo sostenible de la comunidad Kácha'bli (Tesis de Maestría Académica). Programa de Desarrollo Sostenible, con énfasis en Conservación de los Recursos Biológicos, Ciudad Universitaria Carlos Monge Alfaro, Sede de Occidente, Universidad de Costa Rica, San Ramón, Alajuela, Costa Rica.

RAE (Real Academia Española). (2019). Diccionario de la lengua española. Recuperado de https://dle.rae.es/?id=RWmKD5V

Rojas-Morales, Y. (2019, 24 de abril). La universidad no debe considerarse nunca "salvadora" de los pueblos indígenas. Semanario Universidad, pp. 27. Recuperado de https://semanariouniversidad.com/opinion/la-universidadno-debe-considerarse-nunca-salvadora-de-los-pueblos-indigenas/

Taylor, S. J., \& Bogdan, R. (1987). Introducción a los métodos cualitativos de investigación: La búsqueda de los significados. Traduc. J. Piatigorsky. $2^{\text {da. }}$ edic. Barcelona, España: Ediciones Paidós.

Zamora, N. (2010). Fabaceae. En Hammel, B. E., Grayum, M. H., Herrera, C., \& Zamora, N. (Eds.), Manual de Plantas de Costa Rica, vol. V (Clusiaceae-Gunneraceae) (pp. 395-775). St. Louis, Missouri, EE.UU.: Missouri Botanical Garden Press.

\section{APÉNDICE DIGITAL}

Apéndice Digital 1 disponible en archivos suplementarios:

https://revistas.uned.ac.cr/index.php/cuadernos/article/view/2840

Fotografías de algunas de las especies del Cuadro 1. Cada fotografía con el nombre científico de la especie, el nombre de la parte observada y el número de recolecta (PZ\#) 